\title{
The Regulation of LexA on UV-Induced SOS Response in Myxococcus xanthus Based on Transcriptome Analysis
}

\author{
Duo-hong Sheng ${ }^{\# *}$, Ye Wang ${ }^{\#}$, Shu-ge Wu, Rui-qin Duan, and Yue-zhong Li* \\ State Key Laboratory of Microbial Technology, Institute of Microbial Technology, Shandong University, Qingdao \\ 266237, P.R. China
}

\begin{abstract}
SOS response is a conserved response to DNA damage in prokaryotes and is negatively regulated by LexA protein, which recognizes specifically an "SOS-box" motif present in the promoter region of SOS genes. Myxococcus xanthus DK1622 possesses a lexA gene, and while the deletion of lexA had no significant effect on either bacterial morphology, UV-C resistance, or sporulation, it did delay growth. UV-C radiation resulted in 651 upregulated genes in $M$. xanthus, including the typical SOS genes lexA, recA, uvrA, recN and so on, mostly enriched in the pathways of DNA replication and repair, secondary metabolism, and signal transduction. The UV-irradiated lexA mutant also showed the induced expression of SOS genes and these SOS genes enriched into a similar pathway profile to that of wild-type strain. Without irradiation treatment, the absence of LexA enhanced the expression of 122 genes that were not enriched in any pathway. Further analysis of the promoter sequence revealed that in the 122 genes, only the promoters of recA2, lexA and an operon composed of three genes ( $p a f B$, pafC and cyaA) had SOS box sequence to which the LexA protein is bound directly. These results update our current understanding of SOS response in $M$. xanthus and show that UV induces more genes involved in secondary metabolism and signal transduction in addition to DNA replication and repair; and while the canonical LexA-dependent regulation on SOS response has shrunk, only 5 SOS genes are directly repressed by LexA.
\end{abstract}

Keywords: M. xanthus, LexA, SOS response, UV-C irradiation

Received: March 26, 2021 Accepted: May 21, 2021

First published online: May 24, 2021

${ }^{*}$ Corresponding authors Y.Z. Li Phone: +86-532-58631539 E-mail: lilab@sdu.edu.cn D-h. Sheng

Phone: +86-532-58631538 E-mail: dhsheng@sdu.edu.cn

"Duo-hong Sheng and Ye Wang are co-first authors.

Supplementary data for this paper are available on-line only at http://jmb.or.kr.

pISSN 1017-7825 eISSN 1738-8872

Copyright(C) 2021 by The Korean Society for Microbiology and Biotechnology

\section{Introduction}

Genomic DNA is the most important cellular component of bacteria, and its stability is related to bacterial life, death or mutation. However, DNA is vulnerable to a variety of environmental factors such as heat, acidity, dryness, radiation, alkylating agents, free radicals, etc. In response to these DNA threats, bacteria have evolved a conserved inducible DNA damage protection and repair mechanism termed SOS response [1, 2]. The canonical SOS response is known to be LexA/RecA dependent. LexA protein binds specifically to an imperfect palindrome sequence named SOS box (LexA box), which is located on the promoter of SOS genes and blocks their expression. Upon DNA damage, RecA polymerizes along the exposed single-stranded DNA, forming activated nucleoprotein filaments to activate the self-cleavage (autoproteolysis) of LexA, thereby releasing the expression of SOS genes [25]. LexA-dependent SOS response is a unique regulation mode of DNA damage stress response in bacteria and is highly conserved throughout the bacterial domain, making it a potential antibiotic screening target [4-7].

In addition to the ubiquitous LexA-dependent SOS response, LexA-independent pathways have also been found in bacteria to control SOS response [8,9]. For example, in E. coli, the $\beta$-lactam antibiotics that inhibit cellwall synthesis can induce SOS response via a pathway involving the DpiBA two-component signal transduction system [10, 11]. Similarly, E. coli stationary-phase sigma factor RpoS also positively controls the expression of SOS-regulated $\operatorname{din} B$ gene against $\beta$-lactam antibiotics [10-12]. Moreover, Acinetobacter baylyi lacks the lexA gene and the SOS response can be induced in a LexA-independent pathway to activate the DNA repair, in which protein UmuDAb performs like a LexA-like repressor functioning on a sub-set of DNA damage-induced genes [12, 13].

Genome-scale approaches have greatly improved our overall understanding of the SOS genes induced by DNA damage in both LexA-dependent and -independent modes. The number and types of genes regulated by LexA have been found to vary extensively among bacteria. For instance, in UV-irradiated E. coli cells, 168 genes were upregulated of their expression levels, of which 43 genes were directly dependent upon the LexA regulation [14], including genes involved in DNA replication and repair. In the gram-positive model bacterium Bacillus subtilis, the LexA regulon comprises 33 genes among which only eight are homologous counterparts of the E. coli SOS genes [15]. Comparison of the SOS regulons among various bacterial species and genera allowed the description of LexA-regulated core regulons comprising $r e c A, u v r A$, lex $A$ and $\operatorname{rec} N$, which might correspond to the ancestral 
core set [16]. In addition, some genes have been found to be regulated lexA-dependently and -independently [10, 17]. So far, there is no reliable explanation for the evolution and relationship between the manner of LexAdependent and -independent SOS induction.

Myxobacteria are unique among bacteria, being characterized by gliding motility, predation, multicellular fruiting bodies, prolific production of secondary metabolites, and a large genome with many gene duplications. Myxococcus xanthus DK1622 is the model strain of myxobacteria. Previous studies have reported that SOS response in $M$. xanthus could be induced by DNA damage agents (naphthenic acid, mitomycin C or UV ray) [1820 ], but has not been analyzed at the whole genome level. $M$. xanthus contains one lexA gene and two recA genes in its genome and RecAl could facilitate LexA self-cleavage [20]. However, some conserved SOS genes in M. xanthus, including $r e c A 1, u v r A$ or $r e c N$, have no SOS box on their promoters [18]. In order to clarify the SOS response and LexA-dependent regulation in M. xanthus, we compared the transcriptional profiles between the wild type and lexA deletion mutant with UV-C irradiation or not. It was found that UV-C irradiation induced a large number of genes, which were mainly enriched in pathways of DNA replication and repair, metabolism and signaling, and only $5 \mathrm{UV}$-induced genes, including recA2, lexA, pafB, pafC and cyaA, were regulated by LexA directly.

\section{Materials and Methods}

\section{Bacterial Strains and Growth Conditions}

Strains, plasmids, and oligonucleotides used in this study are listed in Tables S6 and S8. The M. xanthus strains were cultivated in Casitone-based CYE medium ( $10 \mathrm{~g} / \mathrm{l}$ casitone, $5 \mathrm{~g} / \mathrm{l}$ yeast extract, $10 \mathrm{mM}$ MOPS and $4 \mathrm{mM}$ $\mathrm{MgSO}_{4}, \mathrm{pH}$ 7.6) for the growth assay or on TPM agar plate for the development assay. E. coli strains were routinely grown on Luria-Bertani (LB) agar or in LB liquid broth $(10 \mathrm{~g} / \mathrm{l}$ peptone, $5 \mathrm{~g} / \mathrm{l}$ yeast extract, and $5 \mathrm{~g} / \mathrm{l} \mathrm{NaCl}, \mathrm{pH} 7.2)$. M. xanthus strains were incubated at $30^{\circ} \mathrm{C}$, and $E$. coli strains were at $37^{\circ} \mathrm{C}$. When required, a final concentration of $33 \mu \mathrm{g} / \mathrm{ml}$ of kanamycin (Kan) or $100 \mu \mathrm{g} / \mathrm{ml}$ of ampicillin (Amp) was added to the solid or liquid media.

\section{Gene Deletion Mutation}

Markerless mutation was carried out to knockout the lexA gene in M. xanthus DK1622 using pBJ113 plasmid. The plasmid contains a kanamycin-resistant cassette for the first round of screening and a galK gene for the negative screening $[32,33]$. To avoid the potential effect on the downstream gene expression, we deleted the

A

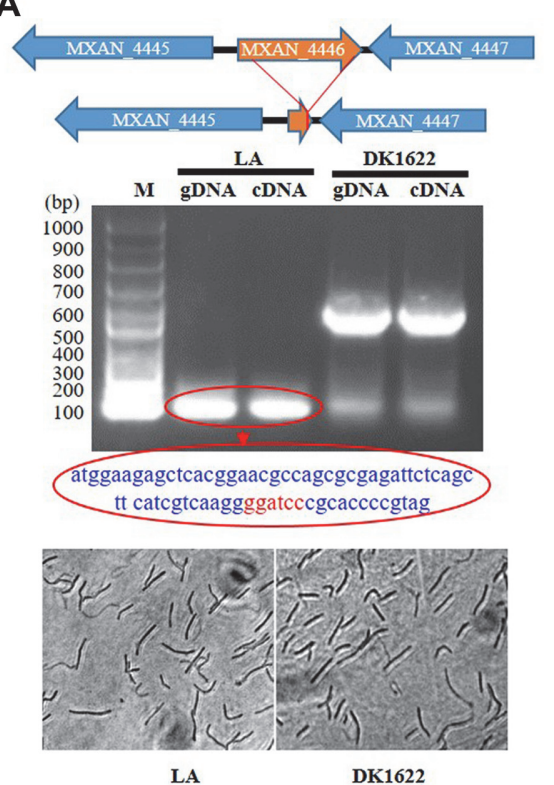

B

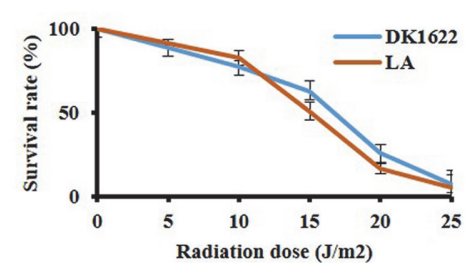

C
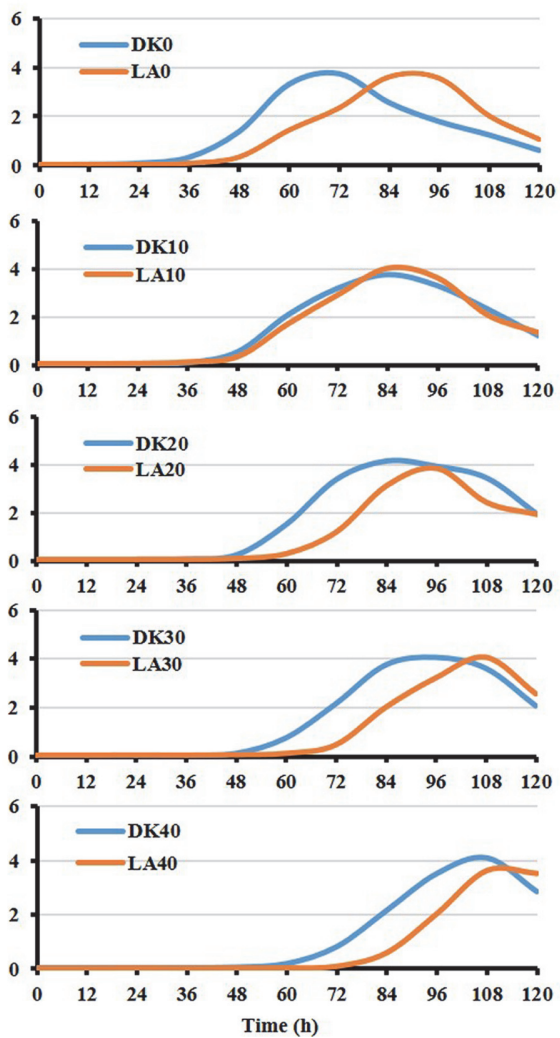

D

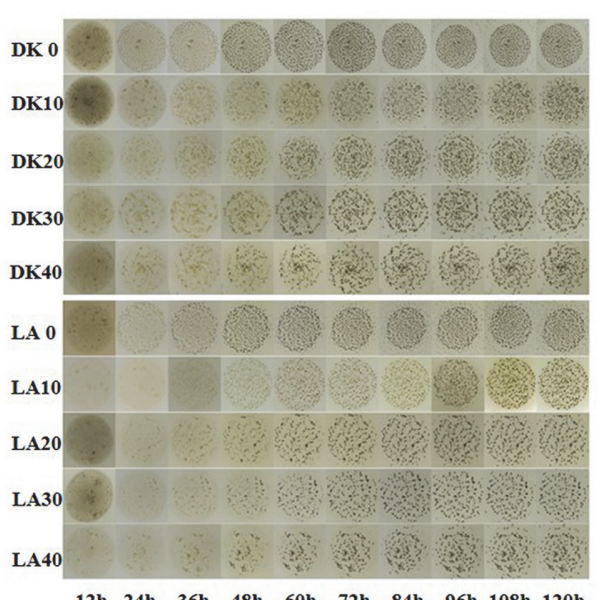

E

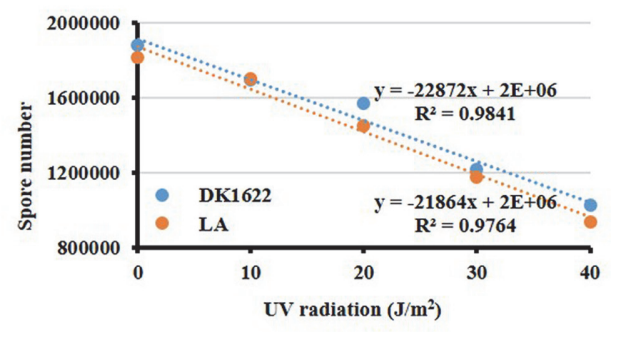

Fig. 1. Deletion mutation analysis of the lexA gene. (A) Construction and verification of the lexA deletion mutant; (B) Survival analysis with different doses of UV-C irradiation in DK1622 and LA; (C) Growth analysis of DK1622 and LA with different doses of UV-C irradiation $\left(0 \sim 40 \mathrm{~J} / \mathrm{m}^{2}\right)$; (D) Fruiting body formation with different doses of UV-C irradiation; (E) Statistical analysis of the sporulation abilities of DK1622 and LA. 
middle part of the lexA gene and left the $\mathrm{N}$ terminal 30-bp and C terminal 9-bp fragments (Fig. 1A). Briefly, both the upstream and down stream homologous arms were amplified with primers (listed in Table S7) and ligated at the BamH1 site. The arm sequences were inserted into the EcoRI/HindIII site of pBJ113. The resulting plasmid was introduced into $M$. xanthus strains via electroporation $(1.25 \mathrm{kV}, 200 \mathrm{~W}, 25 \mu \mathrm{F}, 400 \Omega, 1 \mathrm{~mm}$ cuvette gap). The second round of screening was performed on CYE plates containing 1\% galactose (Sigma). The lexA mutant (named LA) was identified and verified by PCR amplification and sequencing.

Restriction enzymes, DNA ligase, and other enzymes were used according to the manufacturers' recommendations. All DNA products were validated by DNA sequencing.

\section{Growth, Mutation Rate and Sporulation Assays}

M. xanthus cells in exponential phase were used for determining the cell growth curves. For UV irradiation, the cell suspension $(15 \mathrm{ml})$ in $130 \mathrm{~mm}$ glass Petri dishes was stirred gently with a magnetic rod and irradiated with UV ray $(\mathrm{UV}-\mathrm{C}, 254 \mathrm{~nm})$ at room temperature. Then, the cells were diluted in fresh medium and incubated at $30^{\circ} \mathrm{C}$. The density of the cells was measured every $12 \mathrm{~h}$ to generate the growth curves.

The mutation rate assay was conducted by screening the nalidixic acid-resistant strains according to the previous report [34]. Briefly, approximately $5 \times 10^{9}$ cells of $M$. xanthus were placed on CYE agar containing $40 \mu \mathrm{g} / \mathrm{ml}$ nalidixic acid. Nalidixic acid-resistant candidates were counted and mutation rate was calculated using the following formula: mutation rate $=$ nalidixic acid resistant clones $/ 5 \times 10^{9}$.

Sporulation experiments were performed on TPM agar plates [35]. Ten microliters of $\left(10^{7} \mathrm{cells} / \mathrm{ul}\right)$ cells were dot-inoculated on TPM agar plate and allowed to develop at $30^{\circ} \mathrm{C}$. Microscopic examination of cell aggregation and fruiting body formation were conducted every $12 \mathrm{~h}$. Spores were counted after five days as follows: five colonies were collected and serially diluted in liquid CYE medium and myxospores were resuspended by sonication at $200 \mathrm{~W}$ for $10 \mathrm{~s}$ twice. The suspensions were incubated at $55^{\circ} \mathrm{C}$ for $2 \mathrm{~h}$ to kill the vegetative cells and then used for CYE plate numbering.

\section{RNA Extraction, RT-PCR and RNA Sequencing Assay}

Total RNA of $M$. xanthus cells was extracted using RNAiso Plus reagent (Takara, China) following the manufacturer's protocol. RNA concentration was quantified by Nanodrop 2000 (Thermo Fisher Scientific, USA). Genomic DNA (gDNA) in the RNA sample was removed with gDNA Eraser (Takara). The first-strand cDNA was synthesized with random primers by using the PrimeScript RT Enzyme Reagent Kit (Takara) following the manufacturer's instructions.

Synthesized cDNA samples were diluted 5 times prior to RT-PCR. The primers designed for $i m u A$, dnaE2, recA 1 and recA 2 are listed in Table S7. RT-PCR was accomplished using the SYBR Premix Ex Taq Kit (Takara) on an ABI StepOnePlus Real-Time PCR System (Thermo Fisher Scientific), following the program: 3 min at $95^{\circ} \mathrm{C}$, followed by 40 cycles of $30 \mathrm{~s}$ at $95^{\circ} \mathrm{C}, 30 \mathrm{~s}$ at $55^{\circ} \mathrm{C}$, and $15 \mathrm{~s}$ at $72^{\circ} \mathrm{C}$. The relative quantification of mRNAs of interest was performed by the comparative CT $\left(2^{-\Delta \Delta \mathrm{CT}}\right)$ method, with the gapA gene, encoding for glyceraldehyde-3phosphate dehydrogenase, as an endogenous control, as previously described (Peng et al., 2017).

RNA sequencing was conducted by Illumina platform, and the purified double-stranded cDNA was endrepaired, added with A bases, and ligated with sequencing adaptors. After PCR amplification, the cDNA was sequenced on an Illumina HiSeq 4000 (Novogene, China). Three independent repeats were tested for each strain, and the quality information of sequencing for the total 12 samples is shown in Fig. S4. All the upregulated and downregulated genes were obtained by comparing with control, and their gene functions were explored using database annotations such as GO and KEGG. A $p$-value of $<0.05$ was used as the threshold to identify differential expression genes (DEGs).

The level of mRNA expression was normalized by FPKM (fragments per kilobase of transcript per million mapped reads). Genes with a $\log _{2}$ FoldChange $\geq 0.4$ and $p<0.05$ were considered as DEGs.

\section{Protein Expression, Purification and Gel Shift Analysis}

LexA gene was cloned into $E$. coli expression vector $\mathrm{pET} 15 \mathrm{~b}$. Then, the recombinant plasmids were transformed into E. coli BL21(DE3) and cultured in LB medium at $37^{\circ} \mathrm{C}$. Induction of expression was initiated at $\mathrm{OD}_{600}=0.6$ by the addition of IPTG $(1 \mathrm{mM})$. Cells were harvested $3 \mathrm{~h}$ later and sonicated. From crude extracts, the $6 \mathrm{xHis}$-tagged proteins were purified using Ni-NTA agarose columns (Qiagen) according to the manufacturer's instructions.

Gel shift analysis was used to evaluate the binding ability of LexA protein to the SOS box sequence. The SOS box sequence from the primers of genes recA2 (TTTTTTTCGCGTACTAAAAGCGCGTTCAGGTGAGC), lexA (TTTTTTGTTGACTCTACACGTCTGTTCAGGGAGAG), recN (TTTTTTGTCGGACCTGCTAGGGTGTTC AGGTACCA), pafBC (TTTTTTTGATTACCTGCTTGTTTGTTCAGGCGCGT), were synthesized in (TSINGKA) and labeled with biotin at the 5' end. Then, $25 \mu \mathrm{l}$ reactions containing $25 \mathrm{mM}$ Tris- $\mathrm{HCl}, \mathrm{pH} 7.0,50 \mathrm{mM} \mathrm{NaCl}, 4 \%$ glycerol, $1 \mathrm{mM}$ DTT, $2 \mu \mathrm{g}$ protein and $25 \mu \mathrm{mol}$ DNA were incubated at $32^{\circ} \mathrm{C}$ for $20 \mathrm{~min}$, and analyzed by gel electrophoresis in $0.8 \%$ agarose gels. Migration was performed for $2 \mathrm{~h}$, at $2 \mathrm{~V} / \mathrm{cm}$ and $4^{\circ} \mathrm{C}$, and then transferred to a nylon membrane. Blotting was performed using a Bio-Rad electro-blotting system (model Trans blot) according to the manufacturer's instructions. In addition, the biotin-labeled DNA was detected by LightShift Chemiluminescent EMSA Kit (Thermo) according to the instruction manual. Three independent experiments were performed, and a representative sample is shown. 


\section{Results and Discussion}

Absence of lexA Delayed the Growth, But Did Not Affect UV-C Resistance and Sporulation of M. xanthus

There is a single lexA gene in the genome of $M$. xanthus. We deleted most of the lexA gene sequence, leaving only 53 upstream bases and 9 downstream bases (Fig. 1A), and the deletion mutant was named LA. The cell morphology of the mutant did not show filamentous cells like that of the lexA mutation in Clostridium difficile [21]. Using genomic DNA or the cDNA reverse-transcribed from the total RNA as templates, no complete lexA gene was amplified by PCR from the lexA mutant, except for a 70-bp fragment, which was confirmed by sequencing (Fig. 1A). The absence of lexA had little effect on the UV-C resistance of $M$. xanthus cells (Fig. 1B), but delayed the growth of this strain (Fig. 1C). Compared with the wild type, the lexA mutant showed a prolonged lag phase (from 24 to $36 \mathrm{~h}$ ). UV-C radiation treatment further prolonged the lag phase of both the mutant and wildtype strains, which was attributed to DNA damage and cell death. It is noteworthy that the mutant shows more resistance to UV-C radiation at a low dosage $\left(10 \mathrm{~J} / \mathrm{m}^{2}\right)$ than that of wild-type strain (Figs. 1B and $1 \mathrm{C}$ ), which has been thought to be caused by the release of SOS response $[4,5]$.

In the sporulation process, both DK1622 and LA showed cell aggregation to form visible fruiting body at $24 \mathrm{~h}$, and there was no significant difference in their fruiting body formation and sporulation. When the strains were UV-C irradiated, the aggregation abilities of both DK1622 and LA were delayed. With the increase of radiation dose, the visible fruiting bodies and the final number of spores were also decreased gradually and correspondingly with almost no difference in DK1622 and LA treated with the same dose of radiation (Figs. 1D and 1E).

According to the previous reports on other bacteria, the lexA deletion mutant has a distinct SOS-response phenotype, such as inhibited cell division and sporulation, increased UV-C resistance and mutation rate [21-24]. However, the lexA gene deletion in M. xanthus has no significant effect on bacterial morphology, UV-C resistance, and sporulation, but showed delayed growth.

\section{Transcriptional Profiles of UV-Induced M. xanthus}

We inspected the SOS response by RNA sequencing in the UV-C irradiated $M$. xanthus with $15 \mathrm{~J} / \mathrm{m}^{2}$, at which UV significantly affected the survival rates and growth curves of both wild type and mutant (Fig. 1). A total of 1,439 differentially expressed genes (DEGs) were identified in UV-irradiated M. xanthus, including 651 upregulated DEGs and 788 downregulated DEGs (Fig. S1A).

The UV-induced genes (651 upregulated DEGs) were gene-ontology (GO) enriched into 47 functional items ( $p$-values < 0.05) (Fig. S1B), divided into three independent categories: biological process (BP) containing 26 items, accounting for $55.3 \%$ of the total items; cellular component (CC) containing 2 items (4.3\%); molecular function (MF) containing 19 items (40.4\%). There were 8 GO items with more significantly enriched genes $(>30$ DEGs), including $4 \mathrm{BP}$ items (cellular component organization, cellular component organization or biogenesis, DNA metabolic process, and nucleic acid metabolic process) and $4 \mathrm{MF}$ items (heterocyclic compound binding, organic cyclic compound binding, DNA binding, and nucleic acid binding). On the other hand, the 788 downregulated DEGs were enriched in $39 \mathrm{GO}$ items of BP (19 items), CC (7 items) and MF (13 items) groups (Fig. S1C). The most enrichment in $8 \mathrm{GO}$ items (>30 DEGs in each item), were membrane, transporter activity,

A

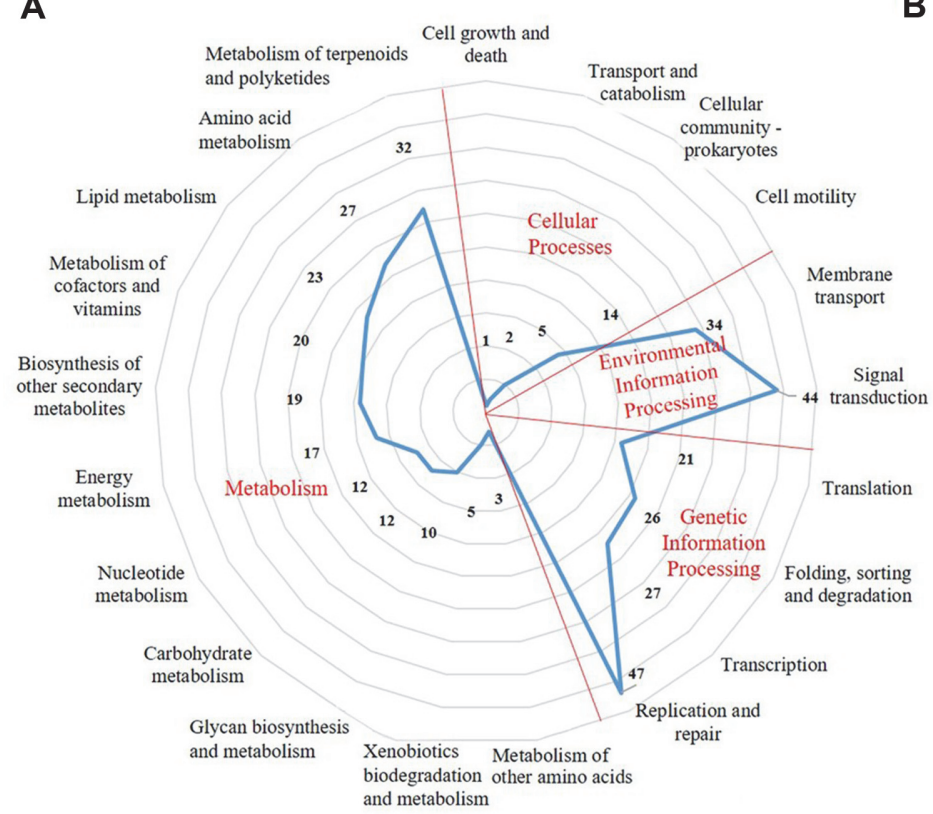

B

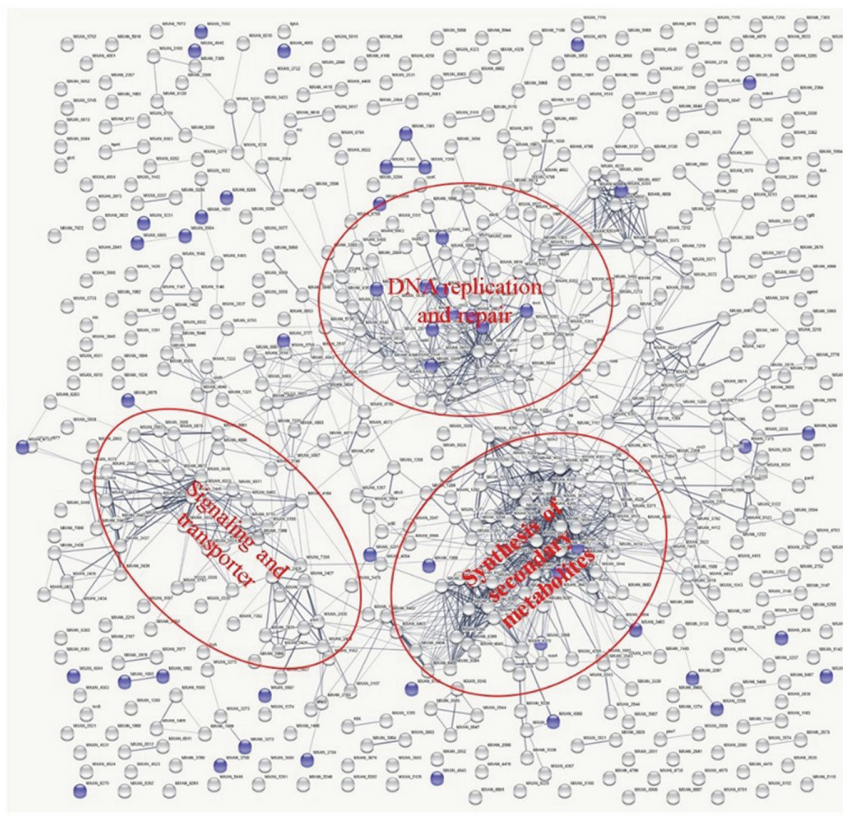

Fig. 2. Functional classification of the UV-induced genes. (A) Gene distribution according to KEGG pathways. (B) Functional protein association networks using STRING. The DEGs affected by LA are shown in blue in B. 
intracellular membrane bound organelle, transmembrane transporter activity, localization, establishment of localization and oxidation-reduction process.

Furtherly, KEGG pathway enrichment analysis of the UV-induced genes led to identification of 21 statistically enriched pathways (Fig. 2A), distributed in metabolism, genetic information processing, environmental information processing, and cellular processes. Obviously, the UV-induced genes were enriched in three pathways of replication and repair, signal transduction, and metabolism of terpenoids and polyketides.

Bacterial SOS response is characterized by the induction of the genes related to DNA damage and repair $[1,2,4]$. In $M$. xanthus, the DNA replication and repair pathway had the largest number of the UV-induced genes

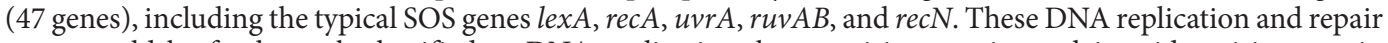
genes could be further sub-classified to DNA replication, base excision repair, nucleic acid excision repair, mismatch repair, recombination repair, and DnaE2-catalyzed TLS processes (Table S1). However, compared with that in E. coli [25], the number of DNA replication and repair genes induced by UV-C radiation decreased in $M$. xanthus. Some known bacterial UV-induced genes were not induced in $M$. xanthus, such as recF, $r e c O, r e c R$, $r v r C, m u t Y, f t s K, r u v A$, and ssb.

The second pathway of gene enrichment was metabolism of terpenoids and polyketides, which is known to be related to secondary metabolites. Using antiSMASH [26], 802 genes involved in 23 secondary metabolite biosynthetic gene clusters (BGCs) were identified in M. xanthus DK1622, of which 217 genes were affected by UV$\mathrm{C}$ radiation and distributed in all the 23 gene clusters, including 126 upregulated genes and 91 downregulated genes (Table S2). The 126 upregulated secondary metabolism related genes were mainly distributed in metabolisms of terpenoids and polyketides, amino acids and lipids, and metabolism of cofactors and vitamins, which enrich most of the UV-induced metabolic genes (Fig. 2A).

The third pathway of gene enrichment was signal transduction of environmental information processing. $M$. xanthus contains many genes involved in signal transduction pathways, including the signal pathways for SOS response [18]. The UV-induced 44 signal transduction genes and 34 membrane transport genes formed the enrichment region of environmental information processing. So many signal proteins are involved in the SOS response, which is helpful to understand the mechanism of LexA-independent SOS induction in M. xanthus.

Meanwhile, we used STRING to search for functional protein association networks of the UV-induced genes [27], and 27 clusters were significantly enriched (Table S3). These local network clusters can be divided into three functional enrichment regions, secondary metabolite, DNA replication and repair, signaling and transporter (Fig. 2B), which is consistent with the functional areas formed by the KEGG pathway enrichment in Fig. 2A.

\section{Transcriptional Profiles of the UV-Irradiated lexA Mutant}

UV-C irradiation treatment on the lexA mutant (LA) resulted in 1,086 DEGs (Fig. S2A), including 412 upregulated DEGs and 674 downregulated DEGs. The induced genes included those conservative SOS genes (recA, lexA, recN, uvrA, and so on), which suggested that the absence of LexA does not affect the induction of SOS response.

Comparing the DEGs between LAUV vs LA and DKUV vs DK1622 (Fig. S2B), 376 DEGs were consistent, and 710 DEGs in LA were inconsistent with those in DK1622. In the upregulated 412 genes of UV-irradiated LA, 289 were synchronously upregulated in UV-irradiated DK1622, occupying more than 70\% of the total upregulated 412 genes. In the downregulated DEGs, however, only less than $10 \%$ of DEGs (66/674) were consistent. Thus, the difference of DEGs between DK1622 and the LA mutant was mainly due to the downregulated DEGs.

GO analysis showed that the upregulated DEGs in the UV-irradiated LA strain were mainly involved in the acyl transfer groups, DNA damage and repair, similar to that of the wild-type strain. The downregulated DEGs were enriched into cellular glucan metabolic process, electron carrier activity, cellulose metabolic process, catabolic process and other metabolic processes (Table S4), which were obviously different from that of wild type. As a result, the difference of DEGs between UV-irradiated LA and wild-type strains might be due to the indirect effect of the inhibition by LexA on bacterial growth.

According to the KEGG pathways, the UV-induced genes in LA were also enriched in replication and repair, signal transduction, and metabolism of terpenoids and polyketides (Fig. S2C), which were similar to that in wild type. The above results showed that the deletion of lexA had less efficient effect on the expression of SOS-induced genes, but more on the bacterial growth, which is consistent with the mutant analysis (Fig. 1).

\section{LexA Repressor Acts on a Small Number of UV-Induced Genes}

Compared with the expression profile of wild-type strain, the absence of lexA resulted in 181 DEGs in M. xanthus (Fig. 3A, Table S5). Among them, 122 were upregulated and 59 were downregulated, accordant with the expectation of LexA as a repressor of SOS response. The DEGs affected by LexA were enriched into 52 GO items, but only the protein phosphorylation exhibited significant difference $(p<0.05)$ (Fig. 3B). Protein phosphorylation is the main mechanism of signal transduction that enables bacteria to rapidly respond to environmental changes by controlling the functional properties of proteins in response to external stimuli [2830]. Notably, the DEGs affected by LexA were not obviously enriched in any KEGG pathway or clusters in functional protein association networks (Fig. 2B).

Comparing the DEGs between LA vs DK1622 and DKUV vs DK1622, 86 DEGs (approximately $47.5 \%$ of the total 181 DEGs in LA) were consistent, which suggested that these genes were involved in UV-induced SOS response (Fig. 3C). In the upregulated DEGs of these two groups, 55 of 122 up regulated DEGs (45\%) in LA were induced by UV-C in wild type. Meanwhile, 10 of 59 down regulated DEGs (16.9\%) in LA were inhibited in UVirradiated wild-type strain. Thus, LexA might act as an inhibitor in the UV-induced M. xanthus SOS response. 

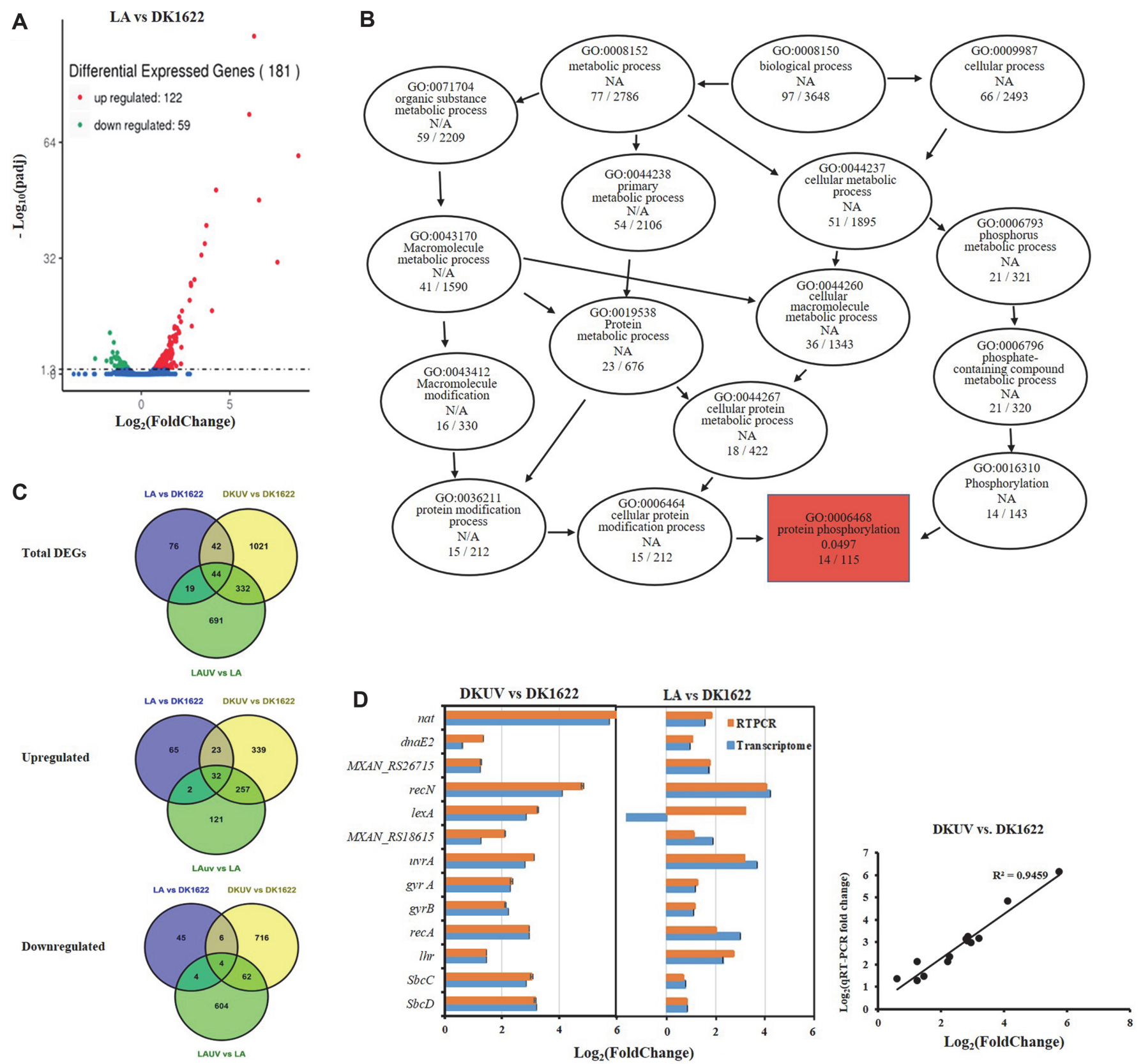

Fig. 3. Analyses of the DEGs from lexA mutant (LA). (A) Volcano plot of DEGs in lexA mutant; (B) GO enrichment in LA; (C) Venn diagram comparison from the combination of LA vs DK1622 and DKUV vs DK1622. (D) RT-PCR verification of 13 differentially expressed DNA replication and repair genes.

In a canonical SOS response, LexA inhibited DNA replication and repair. Thirteen DEGs in the lexA mutant belonged to the DNA replication and repair pathway, of which 12 were UV-upregulated, except lexA. RT-PCR analysis also confirmed the transcriptional change of the 13 genes in lexA mutant (Fig. 3D). The lex $A$ gene was also upregulated in RT-PCR inspection, but downregulated in LA transcriptome, possibly due to the different experimental methods. In transcriptome analysis, the transcriptional sequences of genes are detected and counted. Because most of the coding sequences of lexA gene were missing in the mutant and only the sequences remained at the $\mathrm{C}$ - and $\mathrm{N}$ - terminal of lexA gene could be detected by transcriptome analysis, which showed downregulated expression in the transcriptome of LA. In RT-PCR analysis, the N-terminal retained sequences of lexA gene were used as the detection target which has no difference between wild type and mutant, and the result showed that LexA also repressed its own expression (Fig. 3D).

However, it was true that LexA only affected a fraction of the UV-induced genes, which were scattered and had no obvious function enrichment (marked in blue in Fig. 2B). There were 47 replication and repair genes that were induced by UV-C, and only 13 of them were repressed by LexA and the expression of the other 34 genes was LexA independent (Table S1). Similarly, induction of secondary metabolite synthesis was a characteristic of the UV- 
induced SOS response in M. xanthus, and LexA affected the expression of secondary metabolism genes, but the number of DEGs was significantly less than that induced by UV-C. Compared with the above 217 secondary metabolite synthesis genes affected by UV-C, the deletion of lexA only affected 20 of them (Table S2).

In sum, LexA affects the expression of SOS-induced genes involved in DNA replication and repair and metabolism, but the proportion of SOS genes regulated by LexA is very small among the total of SOS genes.

\section{LexA Directly Inhibited Only Five Regulatory Genes Including Itself}

Comparing the upregulated DEGs between LA vs DK1622 and DKUV vs DK1622 (Fig. 3C), 55 SOS genes (not including lexA) were repressed by LexA, of which 32 could be further upregulated by UV-C in the LA, suggesting that these genes had other regulatory pathways in addition to the LexA-dependent regulation. The other 23 DEGs were not UV-induced in the LA mutant, which probably suggested that they might be affected by LexA only. Interestingly, these 23 genes contained the known classical LexA regulatory genes including $\operatorname{lex} A, \operatorname{rec} N, u v r A$, dnaE2, and recA2.

The binding pattern of $M$. xanthus SOS box (CTRHAMRYBYGTTCAGS) is an imperfect palindrome sequence with the necessary internal bases for LexA binding [18]. We compared the core promoter regions of the 56 LexArepressed DEGs (including lexA gene), and found that only recA2 and lexA had the fully matched SOS box sequences in their core promoter regions (Fig. 4A). The promoter of $r e c N$ has a similar SOS box region with two different bases (CTGCTAGGGTGTTCAGG) from the reported SOS box. In addition, the promoter of pafBC operon, which contains the genes pafB (MXAN_RS06580), pafC (MXAN_RS06585) and cyaA (adenylate cyclase, MXAN_RS06590), also has a similar SOS box (CTGCTTGTTTGTTCAGG).

We expressed and purified the LexA protein (Fig. 4B) and analyzed its binding ability to the four SOS box sequences by gel shift analysis (Fig. 4C). The result showed that that LexA protein could bind the SOS boxes of lexA and recA2 obviously, bind the SOS box of $p a f B C$ weakly, and could not bind the SOS box of recN gene. Furthermore, we searched the whole genome of $M$. xanthus for the SOS box sequence and found five other genes with similar sequences in their promoters, including ferredoxin, heme $\mathrm{ABC}$ exporter $\mathrm{CcmA}$ and three functionunknown genes (Fig. 4A).

Although LexA affected 56 SOS genes, only the expression of $\operatorname{rec} A 2$, lexA, pafB, pafC, and $c y a A$ was directly repressed by LexA. The expression of the other 51 genes was indirectly regulated by LexA. In our previous work, we found that RecA2 was not involved in the DNA recombination and LexA autocleavage, but, instead, in the

A

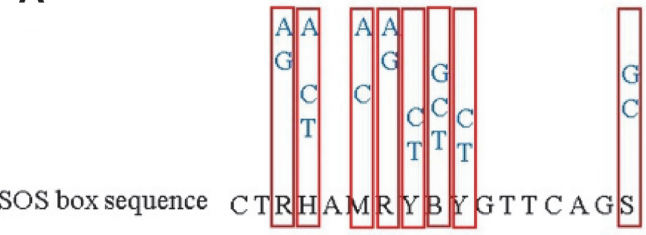

B

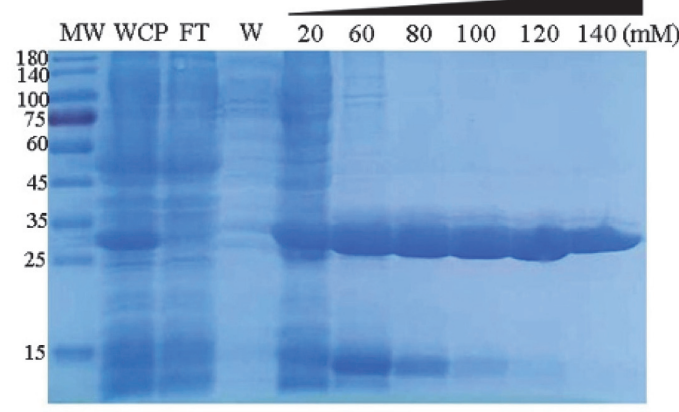

MXAN RS22775 CTACACAGGCGTTCAGG

MXAN_RS02460 C T T G GACGACGT T CA G G

ferredoxin CT TCT ACT GCGT T CAGC

MXAN_RS16510 C T G T GACCACGTTCAGC

CcmA C T TCGCTCCAGTTCAGC

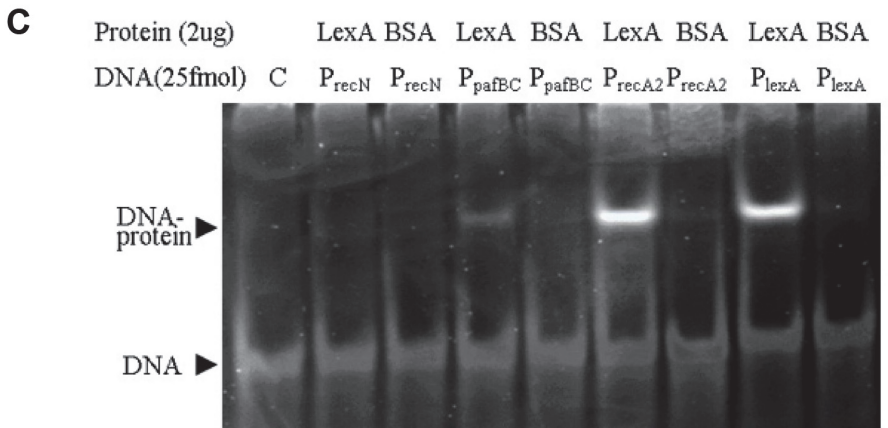

Fig. 4. DNA binding sequences of LexA. (A) Conserved SOS box of LexA regulatory genes. (B) The expression and purification of LexA. (C) Gel shift analysis of LexA binding to the SOS boxes of $r e c N$, recA2, lexA and pafBC. 
regulation on cell growth and antioxidant [20]. Compared with the reported transcriptome data of $r e c A 2$ mutant, 12 of the 51 genes were expressed in a RecA2-dependent manner (as shown in Fig. S3). Similarly, PafBC encoded by $p a f B$ and $p a f C$ was involved in a LexA/RecA-independent DNA damage response [31], and 24 of the 51 genes had the PafBC-binding sequence in their promoters. The adenylyl cyclase encoded by gene cyaA catalyzed the synthesis of cyclic AMP (cAMP), which is one of the most common signaling molecules and is widely distributed from prokaryotes to eukaryotes. Thus, we concluded that LexA has shrunk its regulation on the SOS genes, and the SOS response is more dependent on other signaling pathways. In M. xanthus, LexA does not directly control DNA damage repair related genes, but controls five transcription regulatory protein genes (recA2, lexA, pafB, pafC, and cyaA) to affect the SOS gene expression depending on other signaling pathways (Fig. S3).

\section{Conclusion}

This work is part of the research on the replication, recombination and repair in myxobacteria. UV-C irradiation affected the expression of a large number of genes in M. xanthus, of which 651 genes were up regulated and were mainly distributed in DNA replication and repair, signal transduction, and secondary metabolite synthesis.

The absence of lexA gene in M. xanthus did not show the lexA deletion phenotype common in other bacteria, except for delayed growth. Transcriptome analysis found that the regulatory range of LexA was significantly shrinking in M. xanthus, and the absence of LexA only enhanced the expression of 56 SOS-inducible genes, which was significantly less than the number of SOS genes affected by LexA in E. coli [25]. The 56 genes contained a small part of SOS-induced genes involved in DNA replication and repair and metabolism, but no genes related to sporulation and cell division of Myxococcus. Further LexA binding analysis found that only five SOS-induced genes (recA2, lexA, pafB, pafC, and cyaA) were directly repressed by LexA.

\section{Acknowledgments}

This work was financially supported by the National Natural Science Foundation of China (NSFC) (Nos. 31670076 and 31471183), National Key Research and Development Programs of China (Nos. 2018YFA0900400 and 2018YFA0901704) to YZL and Special National Project on the Investigation of Basic Resources of China (No. 2019FY100700), Key Research \& Developmental Program of Shandong Province (No. 2019JZZY020308) to DHS.

\section{Data Availability Statement}

All datasets generated for this study are included in the manuscript and the supplementary files. The raw sequencing data generated from this study have been deposited in NCBI SRA under the accession number SRP311926.

\section{Conflict of Interest}

The authors have no financial conflicts of interest to declare.

\section{Reference}

1. Radman M. 1975. SOS repair hypothesis: phenomenology of an inducible DNA repair which is accompanied by mutagenesis. Basic Life Sci. 5A: 355-367.

2. Maslowska KH, Makiela-Dzbenska K, Fijalkowska IJ. 2019. The SOS system: a complex and tightly regulated response to DNA damage. Environ. Mol. Mutagen. 60: 368-384.

3. Bertrand-Burggraf E, Hurstel S, Daune M, Schnarr M. 1987. Promoter properties and negative regulation of the uvrA gene by the LexA repressor and its amino-terminal DNA binding domain. J. Mol. Biol. 193: 293-302.

4. Hanawalt PC. 2015. Historical perspective on the DNA damage response. DNA Repair (Amst) 36: 2-7.

5. Butala M, Zgur-Bertok D, Busby SJ. 2009. The bacterial LexA transcriptional repressor. Cell Mol. Life Sci. 66: 82-93.

6. Bellio P, Mancini A, Di Pietro L, Cracchiolo S, Franceschini N, Reale S, 2020. Inhibition of the transcriptional repressor LexA: Withstanding drug resistance by inhibiting the bacterial mechanisms of adaptation to antimicrobials. Life Sci. 241: 117116.

7. Wade JT, Reppas NB, Church GM, Struhl K. 2005. Genomic analysis of LexA binding reveals the permissive nature of the Escherichia coli genome and identifies unconventional target sites. Genes Dev. 19: 2619-2630.

8. Lesca C, Petit C, Defais M. 191. UV induction of LexA independent proteins which could be involved in SOS repair. Biochimie 73: 407-409.

9. Battesti A, Majdalani N, Gottesman S. 2011. The RpoS-mediated general stress response in Escherichia coli. Annu. Rev. Microbiol. 65: 189-213.

10. Miller C, Thomsen LE, Gaggero C, Mosseri R, Ingmer H, Cohen SN. 2004. SOS response induction by beta-lactams and bacterial defense against antibiotic lethality. Science 305: 1629-1631.

11. Pérez-Capilla T, Baquero MR, Gómez-Gómez JM, Ionel A, Martín S, Blázquez J. 2005. SOS-independent induction of dinB transcription by beta-lactam-mediated inhibition of cell wall synthesis in Escherichia coli. J. Bacteriol. 187: 1515-1518.

12. Cook D, Carrington J, Johnson K, Hare J. 2020. Homodimerization and heterodimerization requirements of Acinetobacter baumannii SOS response coregulators UmuDAb and DdrR revealed by two-hybrid analyses. Can. J. Microbiol. 67: 358-371.

13. Hare JM, Perkins SN, Gregg-Jolly LA. 2006. A constitutively expressed, truncated umuDC operon regulates the recA-dependent DNA damage induction of a gene in Acinetobacter baylyi strain ADP1. Appl. Environ. Microbiol. 72: 4036-4043.

14. Courcelle J, Khodursky A, Peter B, Brown PO, Hanawalt PC. 2001. Comparative gene expression profiles following UV exposure in wild-type and SOS-deficient Escherichia coli. Genetics 158: 41-64.

15. Au N, Kuester-Schoeck E, Mandava V, Bothwell LE, Canny SP, Chachu K, 2005. Genetic composition of the Bacillus subtilis SOS system. J. Bacteriol. 187: 7655-7666.

16. Erill I, Campoy S, Barbé J. 2007. Aeons of distress: an evolutionary perspective on the bacterial SOS response. FEMS Microbiol. Rev. 31: 637-656. 
17. Sancar GB, Sancar A, Little JW, Rupp WD. 1982. The uvrB gene of Escherichia coli has both lexA-repressed and lexA-independent promoters. Cell 28: 523-530.

18. Campoy S, Fontes M, Padmanabhan S, Cortés P, Llagostera M, Barbé J. 2003. LexA-independent DNA damage-mediated induction of gene expression in Myxococcus xanthus. Mol. Microbiol. 49: 769-781.

19. Norioka N, Hsu MY, Inouye S, Inouye M. 1995. Two recA genes in Myxococcus xanthus. J. Bacteriol. 177: 4179-4182.

20. Sheng DH, Wang YX, Qiu M, Zhao JY, Yue XJ, Li YZ. 2020. Functional division between the RecA1 and RecA2 proteins in Myxococcus xanthus. Front. Microbiol. 11: 140.

21. Walter BM, Cartman ST, Minton NP, Butala M, Rupnik M. 2015. The SOS response master regulator LexA is associated with sporulation, motility and biofilm formation in Clostridium difficile. PLoS One 10: e0144763.

22. Clerch B, Garriga X, Torrents E, Rosales CM, Llagostera M. 1996. Construction and characterization of two lexA mutants of Salmonella typhimurium with different UV sensitivities and UV mutabilities. J. Bacteriol. 178: 2890-2896.

23. Ramírez-Guadiana FH, Barajas-Ornelas Rdel C, Corona-Bautista SU, Setlow P, Pedraza-Reyes M. 2016. The RecA-dependent SOS response is active and required for processing of DNA damage during Bacillus subtilis sporulation. PLoS One 11: e0150348.

24. Ramírez-Guadiana FH, Del Carmen Barajas-Ornelas R, Ayala-García VM, Yasbin RE, Robleto E, Pedraza-Reyes M. 2013. Transcriptional coupling of DNA repair in sporulating Bacillus subtilis cells. Mol. Microbiol. 90: 1088-1099.

25. Quillardet P, Rouffaud MA, Bouige P. 2003. DNA array analysis of gene expression in response to UV irradiation in Escherichia coli. Res. Microbiol. 154: 559-572.

26. Blin K, Shaw S, Steinke K, Villebro R, Ziemert N, Lee SY, 2019. AntiSMASH 5.0: updates to the secondary metabolite genome mining pipeline. Nucleic Acids Res. 47: W81-W87.

27. Szklarczyk D, Gable AL, Lyon D, Junge A, Wyder S, Huerta-Cepas J, 2019. STRING v11: protein-protein association networks with increased coverage, supporting functional discovery in genome-wide experimental datasets. Nucleic Acids Res, 47: D607-613.

28. Kobir A, Shi L, Boskovic A, Grangeasse C, Franjevic D, Mijakovic I. 2011. Protein phosphorylation in bacterial signal transduction. Biochim. Biophys. Acta 1810: 989-994.

29. Cozzone AJ. 1988. Protein phosphorylation in prokaryotes. Annu. Rev. Microbiol. 42: 97-125.

30. Mijakovic I, Grangeasse C, Turgay K. 2016. Exploring the diversity of protein modifications: special bacterial phosphorylation systems. FEMS Microbiol. Rev. 40: 398-417.

31. Müller AU, Imkamp F, Weber-Ban E. 2018. The mycobacterial LexA/RecA-independent DNA damage response is controlled by pafBC and the pup-proteasome system. Cell Rep. 23:3551-3564.

32. Julien B, Kaiser AD, Garza A. 2000. Spatial control of cell differentiation in Myxococcus xanthus. Proc. Natl. Acad. Sci. USA 97: $9098-$ 9103.

33. Ueki T, Inouye S, Inouye M. 1996. Positive-negative KG cassettes for construction of multi-gene deletions using a single drug marker. Gene 183: 153-157.

34. Tzeng L, Ellis TN, Singer M. 2006. DNA replication during aggregation phase is essential for Myxococcus xanthus development. J. Bacteriol. 2006. 188: 2774-2779

35. Peng R, Chen JH, Feng WW, Zhang Z, Yin J, Li ZS, 2017. Error-prone DnaE2 balances the genome mutation rates in Myxococcus xanthus DK1622. Front. Microbiol. 8: 122. 